\title{
Adaptive Beamforming Using Frequency Invariant Uniform Concentric Circular Arrays
}

\author{
H. H. Chen, Student Member, IEEE, S. C. Chan, Member, IEEE, and K. L. Ho, Senior Member, IEEE
}

\begin{abstract}
This paper proposes new adaptive beamforming algorithms for a class of uniform concentric circular arrays (UCCAs) having near-frequency invariant characteristics. The basic principle of the UCCA frequency invariant beamformer (FIB) is to transform the received signals to the phase mode representation and remove the frequency dependence of individual phase modes through the use of a digital beamforming or compensation network. As a result, the far field pattern of the array is electronic steerable and is approximately invariant over a wider range of frequencies than the uniform circular arrays (UCAs). The beampattern is governed by a small set of variable beamformer weights. Based on the minimum variance distortionless response (MVDR) and generalized sidelobe canceller (GSC) methods, new recursive adaptive beamforming algorithms for UCCA-FIB are proposed. In addition, robust versions of these adaptive beamforming algorithms for mitigating direction-of-arrival (DOA) and sensor position errors are developed. Simulation results show that the proposed adaptive UCCA-FIBs converge much faster and reach a considerable lower steady-state error than conventional broadband UCCA beamformers without using the compensation network. Since fewer variable multipliers are required in the proposed algorithms, it also leads to lower arithmetic complexity and faster tracking performance than conventional methods.
\end{abstract}

Index Terms-Array processing, beamspace, broadband adaptive beamforming, broadband direction-of-arrival (DOA) estimation, frequency invariant, robust beamforming, target tracking, uniform concentric circular arrays (UCCAs).

\section{INTRODUCTION}

B EAMFORMING using sensor arrays is an effective method for suppressing interferences whose angles of arrival are different from the desired looking direction. They find important applications in radio communications, sonar, radar, and acoustics [1]-[3]. Traditional adaptive broadband beamformers usually employ tapped-delay lines or linear transversal filters with adaptive coefficients to generate appropriate beam patterns for suppressing undesirable interferences. Since the response of the array is frequency dependent, the number of coefficients of the tapped-delay lines required will increase with the signal bandwidth. In broadband adaptive beamforming, a considerable number of adaptive coefficients will be required

Manuscript received February 23, 2006; revised January 18, 2007. This paper was presented in part at the IEEE ISCAS' 05 . This paper was recommended by Associate Editor I. Yamada.

H. H. Chen is with the Communication Systems Group, Darmstadt University of Technology, Darmstadt 64283, Germany.

S. C. Chan and K. L. Ho are with the Department of Electrical and Electronic Engineering, The University of Hong Kong, Hong Kong SAR (e-mail: hhchen@eee.hku.hk; scchan@eee.hku.hk; klho@eee.hku.hk.

Digital Object Identifier 10.1109/TCSI.2007.904648 and it translates into increased convergence time and higher implementation complexity. To overcome this problem, subband decomposition technique, partial adaptation, and frequency invariant beamformers (FIBs) [4]-[8] have been proposed to reduce either the frequency band to be adapted or length of the adaptive transversal filters. In FIB, a fixed beamforming network is used to compensate for the frequency dependency of the array and generate beam patterns that are approximately invariant over the frequency band of interest, hence, the name FIBs. There are several techniques for designing FIBs with fixed beampatterns. In [6], the array aperture is discretized to obtain FIB with fixed beampatterns using the scale-frequency relationship of an array aperture. The design and implementation of such FIB for linear arrays have been reported in [7]. Because of the discretization process, the positions of the sensors are usually nonuniform. In [4], it is observed that the design of FIBs for uniform linear arrays (ULA) is equivalent to the design of a 2-D fan filter with different orientations. Using a set of fixed FIBs which cover different spatial angles of a ULA, broadband directional interferences can be suppressed using a generalized sidelobe canceller (GSC)-based [9] adaptive beamformer with very few number of adaptive filter coefficients [5] (one coefficient for each beam). Moreover, the convergence speed is much faster and the steady-state signal to interference ratio is slightly higher than the traditional broadband Griffiths and Jim (GJ) GSC-based adaptive beamformer.

Traditionally, the design of FIBs is mainly focused on linear arrays with fixed spatial-frequency responses [4]-[7], [10]. This is attributed to the attractive linear geometry of the array, which makes the design tractable and enables many efficient direction-of-arrival (DOA) estimation algorithms such as MUSIC [11] to be applied. A related beamspace technique using discrete Fourier transform (DFT), called the frequency-domain frequency invariant beamformers (FDFIBs), for wideband DOA estimation was also reported in [12].

Motivated by the potential advantages of FIB and the symmetric geometry of uniform circular arrays (UCA), Chan and Pun [13] developed an electronic steerable UCA with frequency invariant characteristics. Due to the circular symmetric geometry of the UCA, the frequency invariant array can be obtained by introducing a set of fixed compensated filters [14] and its spatial response is governed by a set of variable coefficients, one per each phase mode.

Unfortunately, the passband of a UCA is closely related to its radius and exhibits a bandpass characteristic. In order to obtain a frequency invariant characteristic over a large bandwidth, the dynamic range of the compensation filters will become very large and it leads to considerable noise amplification of the 
array. This problem can be overcome if uniform concentric circular arrays (UCCA) are employed [15], [16]. Another approach to increase the usable bandwidth of frequency invariant UCA beamformers for microwave applications is to employ directional sensors with appropriate characteristics [17]. Compared to the UCCA proposed in this paper, the directional sensors should possess a frequency invariant beampattern and the radius of the array has to be sufficiently large, while employing fewer number of sensor elements. In [18], it was shown that similar beampatterns of such a UCA can be realized using a two-ring UCCA. Compared with ULAs, UCCAs have the advantages of being able to cover $360^{\circ}$ of the azimuth angle and they can form electronic steerable beampatterns that are relatively frequency invariant over a wide bandwidth. Thus, they are more suitable for 2-D DOA estimation [19] and adaptive beamforming.

In the proposed adaptive UCCA-FIB, signals at the elementspace are first transformed to the phase modes so that the frequency response of the array can be equalized by a set of compensation filters. The compensated phase mode signals are then delayed, multiplied by a set of variable coefficients or weights, and summed to get the beamforming output. Due to the frequency invariant characteristic of the proposed UCCAs, the variable beamforming weight of each phase mode can be adapted as in a conventional narrowband adaptive beamformer using, for example, the minimum variance beamforming (MVB) [20] and GSC [9] to suppress the undesirable interferences. Therefore, the length of the tapped-delay line can be drastically reduced (one per phase mode in our experiment). As a result, the arithmetic complexity associated with the lengthy variable tapped delay lines in traditional broadband adaptive beamformers can be significantly reduced and the convergence speed will also be greatly improved. In [18], the theory, design, DOA estimation, and beamforming using the sample matrix inversion (SMI) method are described.

In this paper, we shall further study the recursive and robust implementation of the adaptive UCCA-FIB using the GSC algorithm and compare its performance with traditional tappeddelay line implementation. Simulation results show that the proposed adaptive UCCA-FIB has much faster convergence speed and much fewer number of adaptive beam weights than conventional broadband adaptive beamformers using UCCAs without the compensation filters. In addition, the performance of the proposed adaptive UCCA-FIB in tracking moving sources is much better than UCCAs using conventional tapped-delay line adaptive beamformers.

Due to DOA estimation and other implementation errors such as calibration errors, the actual array response may derivate from the assumed signal model. As a result, conventional beamforming methods such as GSC may suppress the desired signal as well as the interfering signals, causing signal degradation or even cancellation. Robust beamforming algorithms were proposed to remedy this problem. A conventional approach is to limit the norm of the adaptive weight vector [21], so called norm constraint, to prevent the leakage signal coming from the blocking matrix of the GSC from canceling the desired signal in the fixed desired beam. For recursive least squares (RLS) implementation of the adaptive beamformer, this norm

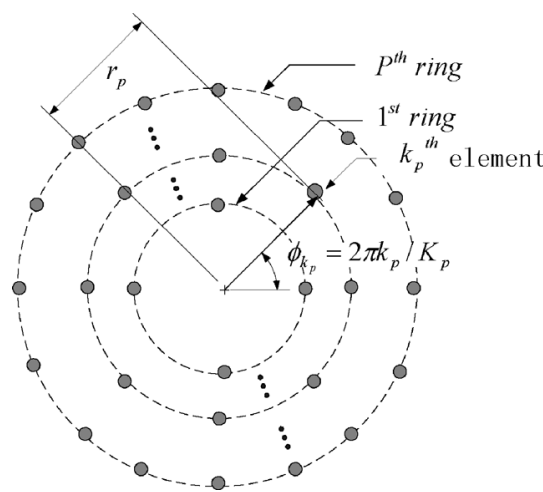

Fig. 1. UCCA with $P$ rings. The $p$ th ring has $K_{p}$ equally spaced sensors.

constraints can also be realized by adding a small identity matrix to the auto-correlation matrix of the sensor output. Because of this operation, this method is also called diagonal loading [22]. More recent approaches formulate the robust beamforming problem as a convex optimization problem where the worst-case performance is optimized subject to certain models of uncertainty [23], [24]. In this paper, we shall develop robust adaptive beamforming algorithms and diagonal loading approaches for UCCA-FIBs because of their good performance and low implementation complexity. The performance of the proposed robust UCCA-FIB is evaluated in target tracking and the presence of DOA estimation error or sensor calibration error. Simulation results show that the robust UCCA-FIBs have a better performance than its origin counterpart when there are sensor location uncertainties. The algorithms proposed in this paper are also applicable to the frequency invariant UCAs as proposed in [13], though they, in general, have a narrower bandwidth than frequency invariant UCCAs.

This paper is organized as follows. In Section II, the structure and the design of the broadband UCCA-FIB will be briefly reviewed. The proposed broadband adaptive beamforming algorithms using the UCCA-FIB are presented in Section III. The robust UCCA-FIB beamforming algorithms are developed in Section IV. The DOA estimation and target tracking algorithms employing the UCCA-FIB are also described. Design examples and simulation results are given to illustrate the usefulness of the proposed methods. Conclusions are drawn in Section V.

\section{FREQUENCY INVARIANT UCCAS}

The structure and the design of the frequency invariant UCCA [15], [16] is briefly reviewed in this section. The geometrical structure of a $P$-ring UCCA is shown in Fig. 1 . There are $K_{p}$ omni-directional elements in each ring and the sensor elements are located along the circumference of a circle according to $\left\{r_{p} \cos \phi_{k}, r_{p} \sin \phi_{k}\right\}$ with the center of the rings situated at the origin of the Cartesian coordinate, where $r_{p}$ is the radius of the $p$ th ring, $p=1, \ldots, P, \phi_{k}=2 \pi k / K_{p}$ and $k=0, \ldots, K_{p}-1$ as shown in Fig. 2. With the sensor distance being fixed at half of the smallest wavelength $\lambda_{\min }$ of the array to be operated, the radius of the $p$ th ring of the UCCA is given by

$$
r_{p}=\frac{\lambda_{\min }}{\left(4 \sin \left(\frac{\pi}{K_{p}}\right)\right)} .
$$




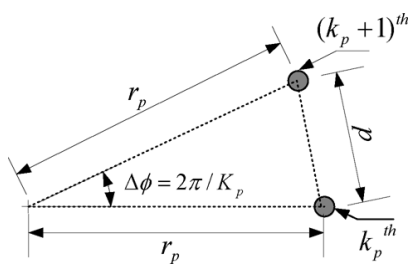

Fig. 2. Relationship between inter-sensor spacing and the radius of the $p$ th ring of the UCCA.

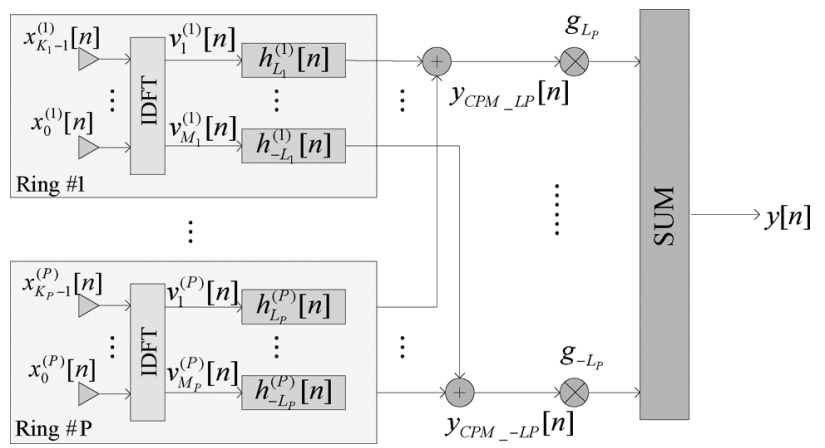

Fig. 3. Block diagram of a $P$-ring UCCA-FIB.

For convenience, this radius is represented as its normalized version: $\hat{r}_{p}=r_{p} / \lambda_{\min }=1 /\left(4 \sin \left(\pi / K_{p}\right)\right)$. The steering vector of the $p$ th ring of a UCCA can be written as

$\mathbf{a}_{p}(\omega, \phi)=\left[e^{j \omega \hat{r}_{p} \alpha \sin \theta \cos \left(\phi-\phi_{0}\right)}, e^{j \omega \hat{r}_{p} \alpha \sin \theta \cos \left(\phi-\phi_{1}\right)}\right.$,
$\left.\ldots, e^{j \omega \hat{r}_{p} \alpha \sin \theta \cos \left(\phi-\phi_{K_{p}-1}\right)}\right]^{T}$

where $j=\sqrt{-1}, \alpha=f_{s} / f_{\max }$ denotes the ratio of the sampling frequency $f_{s}$ to the maximum frequency $f_{\max }, \omega$ is the angular frequency, $\theta$ is the elevation angle that is measured from an imaginary reference axis perpendicular to the horizontal plane, $\phi$ is the azimuth angle measured from an imaginary reference axis on the horizontal plane of the sensors and $\phi_{k}$ is the azimuth angle of the $k$ th sensor. In this paper, we focus our design at an elevation angle of $\theta=\pi / 2$, i.e., the horizontal plane.

The structure of the UCCA frequency invariant beamformer (FIB) is shown in Fig. 3. After appropriate down-conversion, low-pass filtering and sampling, the sampled signals of the $p$ th ring from the antennas are given by the vector $x_{p}[n]=\left[x_{0}^{(p)}[n] x_{1}^{(p)}[n] \cdots x_{K_{p}-1}^{(p)}[n]\right]^{T}$, which is called a snapshot at sampling instance $n$. Each snapshot is inverse discrete Fourier transformed (IDFT) to a set of Fourier coefficients, each coefficient is called a phase mode $V_{m}^{(p)}[n]$, $m=-L_{p}, \ldots, L_{p}, p=1, \ldots, P$. Each phase mode is then filtered or compensated by a compensation filter with impulse response $\boldsymbol{h}_{m}^{(p)}[n]$. The compensated signals are then input to the adaptive beamforming network to form the output of the entire adaptive UCCA-FIB. Since the compensated phase mode signals are relatively frequency invariant in the desired frequency band, very few taps are required in the subsequent adaptive beamforming network for extracting the desired signals and suppressing the interferences. In our simulations, one tap per phase mode is found to give satisfying performance. In other words, the adaptive beamforming network is working like a narrow band adaptive beamformer. We now briefly describe the principle and design of the UCCA-FIB.

The phase mode signals of the $p$ th ring of the UCCA are obtained by IDFT transforming the snapshot samples $x_{p}[n]$

$$
v_{m}^{(p)}[n]=\sum_{k=0}^{K_{p}-1} e^{j\left(2 \pi k m / K_{p}\right)} x_{k}^{(p)}[n], \quad m=-L_{p}, \ldots, L_{p}
$$

$L_{p}=\left(M_{p}-1\right) / 2$ and $M_{p}$ is the number of the phase modes, which is assumed to be an odd number. Each branch of the IDFT output is then filtered by the compensation filter $\boldsymbol{h}_{m}^{(p)}[n]$ with frequency response $H_{m}^{(p)}(\omega)$, to compensate for the frequency dependency of the phase mode. It is then multiplied by the variable beamformer weight $g_{m}^{(p)}$ before combining to give the output of the $p$ th ring $y_{p}[n]$ as follows:

$$
y_{p}[n]=\sum_{m=-L_{p}}^{L_{p}}\left(v_{m}^{(p)} * \boldsymbol{h}_{m}^{(p)}\right)[n] \cdot g_{m}^{(p)}
$$

where $*$ denotes discrete-time convolution. To obtain the spatial-temporal transfer function of the $p$ th ring, let us assume that there is only one source signal $s[n]$ with spectrum $S(\omega)$. Taking DTFT on both sides of (2.4), we have

$$
\begin{aligned}
& Y_{p}(\omega)=S(\omega) \\
& \cdot \sum_{m=-L_{p}}^{L_{p}} g_{m}^{(p)}\left(\sum_{k=0}^{K_{p}-1} e^{j \omega \hat{r}_{p} \alpha \cos \left(\phi-\phi_{k}\right)} e^{j\left(2 \pi k m / K_{p}\right)}\right) H_{m}^{(p)}(\omega)
\end{aligned}
$$

where we have used the fact that the Fourier transform of $v_{m}^{(p)}[n]$ is $V_{m}^{(p)}(\omega)=S(\omega) \cdot \sum_{k=0}^{K_{p}-1} e^{j \omega \hat{r}_{p} \alpha \cos \left(\phi-\phi_{k}\right)} e^{j\left(2 \pi k m / K_{p}\right)}$.

In a UCCA FIB, the outer rings have more phase modes than the inner ones. For simplicity, we let the weighting vectors of the rings be identical, i.e., $\boldsymbol{g}_{m}^{(p)}=\boldsymbol{g}_{m}, p=1, \ldots, P$. The overall output of the array is then obtained by summing the contribution from each ring as given by (2.5). This gives

$$
Y(\omega)=S(\omega) G(\omega, \phi)
$$

where

$$
\begin{aligned}
G(\omega, \phi)= & \sum_{p=1}^{P} \sum_{m=-L_{P}}^{L_{P}} g_{m} \\
& \cdot\left[\sum_{k=0}^{K_{p}-1} e^{j \omega \hat{r}_{p} \alpha \cos \left(\phi-\phi_{k}\right)} e^{j\left(2 \pi k m / K_{p}\right)} H_{m}^{(p)}(\omega)\right]
\end{aligned}
$$

is the spatial-frequency response of the UCCA. To obtain a frequency invariant response, the term inside the bracket, which is a function of both $\phi$ and $\omega$, should be independent of the frequency variable $\omega$. If the number of sensors is large enough, $G(\omega, \phi)$ can be approximated by [18]

$G(\omega, \phi) \approx \sum_{m=-L_{p}}^{L_{p}} g_{m} e^{j m \phi}\left[\sum_{p=1}^{P} K_{p} j^{m} J_{m}\left(\omega \hat{r}_{p} \alpha\right) \cdot H_{m}^{(p)}(\omega)\right]$.

To achieve a frequency invariant response over the desired band, the term inside the bracket of (2.7) should be equal to 1 . 
For a uniform circular array with one single ring in the array, the compensation filters can be chosen as the inverse of the Bessel functions $J_{m}\left(\omega \hat{r}_{p} \alpha\right)$. However, the value of the Bessel function may be zero or nearly zero in the interested frequency band, which may increase considerably the dynamic range of the compensated filter $H_{m}^{(p)}(\omega)$ and consequently lead to considerable noise amplification at the array output. Fortunately, by employing more rings, i.e., UCCA, it can be shown that $H_{m}^{(p)}(\omega)$ 's are required to compensate for the $m$ th phase mode from all the rings. Since these responses, $J_{m}\left(\omega \hat{r}_{p} \alpha\right)$, from different rings cover different frequency bands, i.e., not entirely zero, it is possible to choose compensation filters with small dynamic ranges in all the interested bands for most of the phase modes available. On the other hand, some of the high-order phase modes of the outermost ring do not have any counterparts from other rings and they will be discarded. The number of these usable phase modes will determine the length of $\boldsymbol{g}_{m}$, i.e., $L_{P}$ in (2.7). This will limit the ultimate performance of the spatial beampattern. From (2.7), it can be seen that if the filters $H_{m}^{(p)}(\omega)$ are designed in such a way that

$$
\begin{aligned}
& \sum_{p=1}^{P} K_{p} j^{m} J_{m}\left(\omega \hat{r}_{p} \alpha\right) \cdot H_{m}^{(p)}(\omega) \approx 1, \\
& \qquad \text { for } \omega \in\left[\omega_{L}, \omega_{U}\right], m=-L_{P}, \ldots, L_{P}
\end{aligned}
$$

where $\omega_{L}$ and $\omega_{U}$ are, respectively, the lower and upper frequencies of interest, then the beamformer will be approximately frequency invariant within $\omega \in\left[\omega_{L}, \omega_{U}\right]$ and

$$
G(\omega, \phi) \approx \sum_{m=-L_{P}}^{L_{P}} g_{m} e^{j m \phi} .
$$

From this equation, we can see that the far field beam pattern is now governed by the spatial weighting $\left\{g_{m}\right\}$ alone and it can be written as $G(\omega, \phi) \approx G(\phi)$, which is similar to that of a digital finite duration impulse response (FIR) filter with impulse response $\left\{g_{m}\right\}$. Therefore, the beampattern $G(\phi)$ can be designed separately from the compensation filters by conventional filter design algorithms such as the Parks-McClellan algorithm [26] or second-order cone programming (SOCP), if convex quadratic constraints are to be imposed [27]. Moreover, real-time adaptation of the beam pattern through the spatial weighting $\left\{g_{m}\right\}$ to suppress undesired interferences is also simpler than traditional broadband UCCA adaptive arrays using tapped delay lines as we will see later in Section III.

Since the left-hand side of (2.8) is a linear function of the filter coefficients in $H_{m}^{(p)}(\omega)$ 's, the design problem in (2.8) can be treated as a digital FIR filter design problem with all the filter outputs adding up to the desired response, which is equal to one. If the minimax error criterion is used, the filter coefficients for $H_{m}^{(p)}(\omega)$ can be determined by SOCP [27], [28]. Due to page limitation, we only illustrate the principle and concept of the UCCA-FIB by an example and refer interested readers to [18] for more details.

1) Example 1: UCCA-FIB With Two Rings: In this example, a two-ring UCCA is considered. The inner ring and the outer ring have 10 and 18 omni-directional sensors, respectively. The

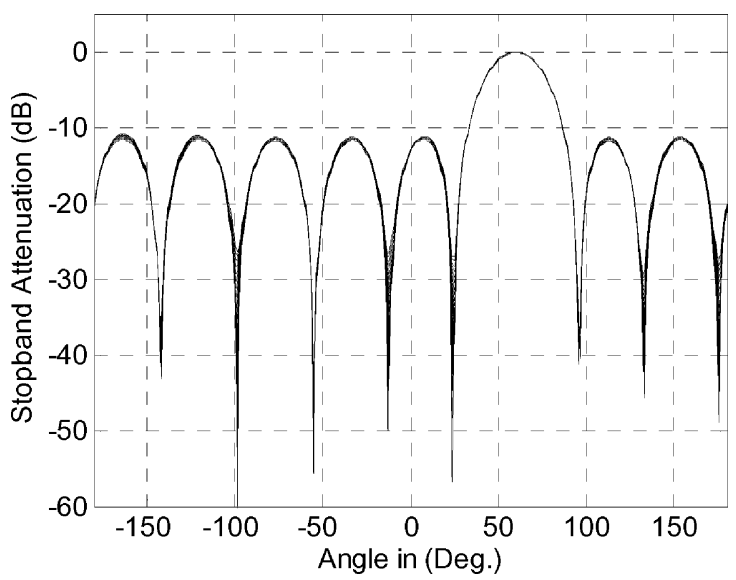

Fig. 4. Spatial response of the UCCA-FIB with two rings.

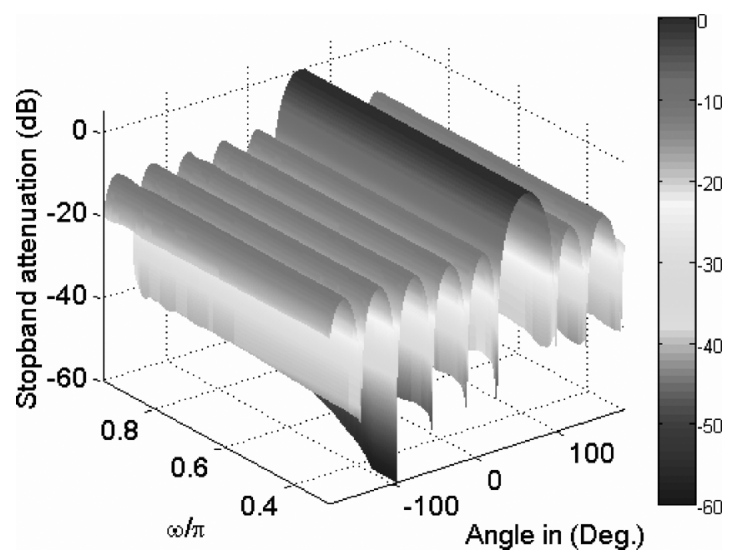

Fig. 5. Spatial-frequency response of the UCCA-FIB with two rings.

required bandwidth of the UCCA- FIB is $\omega \in[0.3 \pi, 0.95 \pi]$. The numbers of phase modes $M$ of the inner and outer rings are 9 and 17, respectively. To avoid noise amplification, 9 out of the 17 phase modes are chosen. The desired beam is targeted at $60^{\circ}$ and the beamwidth is $10^{\circ}$. The magnitudes of the compensation filters in the inner ring and outer ring are constrained to be less than 0 and $26 \mathrm{~dB}$, respectively. $\left\{g_{m}\right\}$ are obtained from the Parks-McClellan algorithm according to the beam direction and width with the same passband and stopband ripples. The frequency responses are shown in Figs. 4 and 5. For convenience, the frequency responses of the UCCA-FIB for $\omega \in[0.3 \pi, 0.95 \pi]$ are discretized with 128 samples and are plotted together in Fig. 4 to compare the array spatial responses at different frequencies. It can be seen that the array responses almost overlap each other, which illustrates that the array response is approximately frequency invariant over the desired band. Fig. 5 shows the perspective view of the beamformer.

\section{AdAPTIVE BEAMFORMING USING UCCA-FIB}

The UCCA-FIB designed in Section II can be used in broadband adaptive beamforming. With the FI characteristic, the length of the variable weight vector in the beamformer can be significantly reduced compared to conventional UCCAs without employing the compensation network. A recursive adaptive beamforming algorithm using the minimum variance 
distortionless response (MVDR) and the GSC is developed in the following.

Consider $I$ broadband signals $\boldsymbol{s}[n]=\left[s_{1}[n], \ldots, s_{I}[n]\right]^{T}$, which impinge a $P$-ring UCCA, respectively, at azimuth angles $\phi_{i}, i=1, \ldots, I$. According to (2.6) and let $\boldsymbol{S}(\omega)=\left[S_{1}(\omega), \ldots, S_{I}(\omega)\right]^{T}$ denote the spectrum of the impinging signal $\boldsymbol{s}[n]$, the output of the UCCA-FIB can be written as

$$
Y(\omega)=\sum_{m=-L_{P}}^{L_{P}} g_{m}\left[\boldsymbol{a}_{G_{m}}^{T}(\omega, \Phi) \boldsymbol{S}(\omega)+N_{m}(\omega)\right]
$$

where $\boldsymbol{a}_{G_{m}}(\omega, \Phi)=\left[a_{G_{m}}\left(\omega, \phi_{1}\right), \ldots, a_{G_{m}}\left(\omega, \phi_{I}\right)\right]^{T}$

$$
a_{G_{m}}\left(\omega, \phi_{i}\right)=\sum_{p=1}^{P} \sum_{k=0}^{K_{p}-1} e^{j \omega \hat{r}_{p} \alpha \cos \left(\phi_{i}-\phi_{k}\right)} e^{j\left(2 \pi k m / K_{p}\right)} H_{m}^{(p)}(\omega)
$$

$i=1, \ldots, I, N_{m}(\omega)=\sum_{p=1}^{P} \sum_{k=0}^{K_{p}-1} e^{j 2 \pi k m / K_{p}} N_{k}^{(p)}(\omega)$ $H_{m}^{(p)}(\omega)$ and $N_{k}^{(p)}(\omega)$ is the frequency response of the additive white Gaussian sensor noise $N_{k}^{(p)}[n]$ at the $k$ th element of the $p$ th ring. From Section II, we know that $\boldsymbol{a}_{G_{m}}(\omega, \Phi)$ is designed to be frequency invariant and hence

$$
\boldsymbol{a}_{G_{m}}(\omega, \Phi) \approx \boldsymbol{a}_{G_{m}}(\Phi), \omega \in\left[\omega_{l}, \omega_{u}\right] .
$$

Also, for notation convenience, we shall replace the approximation sign in (3.2) by the equality sign and assume that the errors are absorbed into the sensor noise. To be consistent with the notation used in the literature, $\boldsymbol{w}=\left[w_{1}, \ldots, w_{M_{P}}\right]^{T}$ will be used instead of the weight vector $\boldsymbol{g}$. Taking the IDFT of (3.1) and using (3.2), one gets the time-domain expression as follows:

$$
\begin{aligned}
y[n] & =\sum_{m=-L_{P}}^{L_{P}} g_{m}\left\{\boldsymbol{a}_{G_{m}}^{T}(\Phi) \boldsymbol{s}[n]+\eta_{m}[n]\right\} \\
& =\boldsymbol{w}^{H} \boldsymbol{a}_{G}(\Phi) \cdot \boldsymbol{s}[n]+\boldsymbol{w}^{H} \boldsymbol{\eta}_{\mathrm{CPM}}[n] \\
& =\boldsymbol{w}^{H} y_{\mathrm{CPM}}[n]
\end{aligned}
$$

where $\boldsymbol{a}_{G}(\Phi)=\left[\boldsymbol{a}_{G_{-L_{P}}}(\Phi), \ldots, \boldsymbol{a}_{G_{L_{P}}}(\Phi)\right]^{T}$ is an $\left(M_{P} \times I\right)$ source direction matrix with $\boldsymbol{a}_{G_{m}}(\omega, \Phi) \approx \boldsymbol{a}_{G_{m}}(\Phi)$, $\boldsymbol{\eta}_{\mathrm{CPM}}[n]=\left[\eta_{-L_{P}}[n], \ldots, \eta_{L_{P}}[n]\right]^{T}$ is the noise vector containing the noises at the compensated phase modes of the beamformer, and

$$
\boldsymbol{y}_{\mathrm{CPM}}[n]=\left[y_{\mathrm{CPM}_{-}-L_{P}}[n], \ldots, y_{\mathrm{CPM}_{-} L_{P}}[n]\right]^{T}
$$

is the compensated phase mode vector with $y_{\text {CPM_ }-m}[n]=$ $\boldsymbol{a}_{G_{m}}^{T}(\Phi) \boldsymbol{s}[n]+\eta_{m}[n]$ be the $m$ th compensated phase mode signal.

Assume that the desired signal impinges the array at an azimuth angle $\phi_{d}$. To recover the desired signal from the array output, the classical MVB [20] (or minimum variance distortionless response MVDR beamformer) is employed in [18]. The basic idea of MVB is to choose the weight vector $\boldsymbol{w}$ such that the output energy of the array is minimized, while requiring the response of the array in the looking direction $\phi_{d}$ to be 1 . The structure of the MVDR beamformer is shown in Fig. 6 . The input of the beamformer is the compensated phase mode

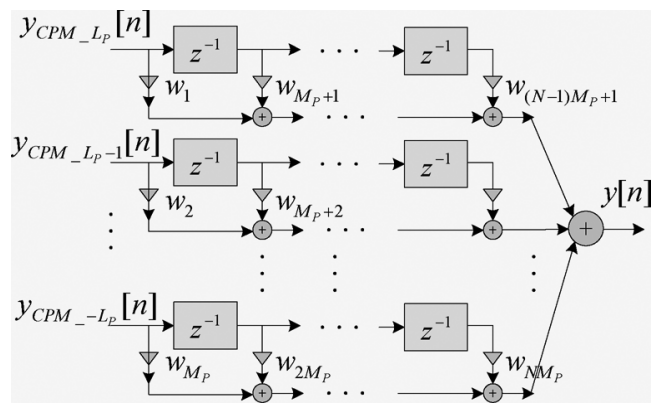

Fig. 6. Structure of the MVDR Beamformer.

vector $\boldsymbol{y}_{\mathrm{CPM}}[n]$, which is delayed and weighted to get the desired signal. Each delay line is a linear transversal filter and is called a tapped-delay line. The MVDR problem is

$$
\min _{w} E\left[|\boldsymbol{y}[n]|^{2}\right] \text { with } \boldsymbol{C}^{H} \boldsymbol{w}=\mathbf{F}
$$

where

$$
\boldsymbol{C}=\left[\begin{array}{ccc}
\boldsymbol{a}_{G}\left(\phi_{d}\right) & & \mathbf{0} \\
& \ddots & \\
\mathbf{0} & & \boldsymbol{a}_{G}\left(\phi_{d}\right)
\end{array}\right]
$$

$\mathbf{F}=[1,0, \ldots, 0]^{T}, \boldsymbol{y}[n]$ is the beamformer output, $\boldsymbol{w}=\left[w_{1}, w_{2}, \ldots, w_{N M_{P}}\right]^{T}$ is the weight vector and its elements are arranged column by column as shown in Fig. 6. $M_{P}$ is the number of the phase modes used in the array and $N$ is the number of taps in each tapped delayline. Substituting (3.3) and (3.4) into (3.5), the MVDR problem can be rewritten as:

$$
\text { minimize } \boldsymbol{w}^{H} \boldsymbol{R}_{y_{-} \mathrm{CPM}} \boldsymbol{w} \quad \text { subject to } C^{H} \boldsymbol{w}=\mathbf{F}
$$

where $\boldsymbol{R}_{y_{-} \mathrm{CPM}}=E\left\{\boldsymbol{y}_{\mathrm{CPM}_{-} D}[n] \cdot \boldsymbol{y}_{\mathrm{CPM}{ }_{-} D}^{H}[n]\right\}$ is the autocorrelation of the data matrix and

$$
\begin{aligned}
y_{\mathrm{CPM}_{-} D}[n]=\left[\boldsymbol{y}_{\mathrm{CPM}}^{T}[n], \boldsymbol{y}_{\mathrm{CPM}}^{T}[n-1],\right. & \ldots, \\
& \left.\boldsymbol{y}_{\mathrm{CPM}}^{T}[n-N+1]\right]^{T} .
\end{aligned}
$$

This constrained optimization can be solved analytically and the optimal solution is

$$
\boldsymbol{w}_{\mathrm{opt}}=\boldsymbol{R}_{y_{-} \mathrm{CPM}}^{-1} \boldsymbol{C}\left(\boldsymbol{C}^{H} \boldsymbol{R}_{y_{-} \mathrm{CPM}}^{-1} \boldsymbol{C}\right)^{-1} \mathbf{F} .
$$

Given a series of snapshots $\boldsymbol{y}_{\mathrm{CPM}}[n]$, say $n=1, \ldots, K$, the auto-correlation matrix can be estimated as $\hat{\boldsymbol{R}}_{y_{-} \mathrm{CPM}}=$ $(1 / K) \sum_{n=1}^{K} \boldsymbol{y}_{\mathrm{CPM}_{-} D}[n] \boldsymbol{y}_{\mathrm{CPM}_{-} D}^{H}[n]$. Thus, $\boldsymbol{w}_{\text {opt }}$ can be obtained by inverting the matrix $\hat{\boldsymbol{R}}_{y_{-} \mathrm{CPM}}$ and substituting it into the right-hand side of (3.7). This is called the SMI method.

The SMI method is computational expensive because it requires the inversion of the autocorrelation matrix. Alternatively, the weight vector $\boldsymbol{w}$ can be solved recursively using adaptive filtering algorithms such as the RLS algorithm and the least mean squares (LMS) algorithm using a structure called GSC [32]. Fig. 7 shows the structure of the GSC beamformer. The weighting vector $w$ is decomposed into two parts: the fixed part $\boldsymbol{w}_{c}$ and the adaptive part $\boldsymbol{w}_{a}$. The fixed weight vector $\boldsymbol{w}_{c}$, as 


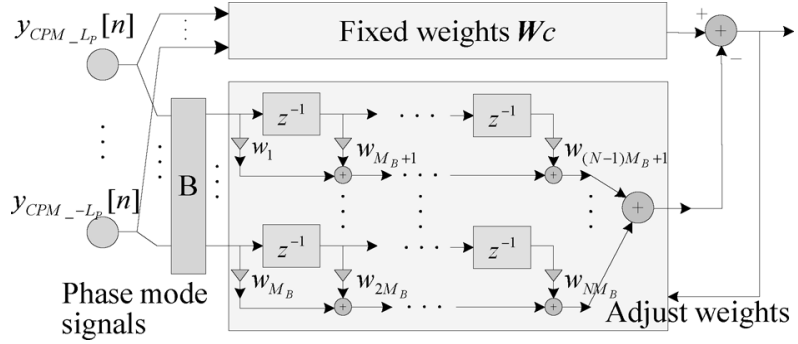

Fig. 7. Structure for GSC beamformer.

shown in the upper part in Fig. 7, forms a main beam that is steered towards some assumed propagation direction. Normally, the beam is designed to have a looking direction at zero degree and the desired looking direction is obtained by delaying the sensor inputs appropriately. In the proposed UCCA-FIB, since the direction of the beam can be readily changed by modulating the beam weight $\left\{g_{m}\right\}$ with an appropriate sinusoid, these delay elements are unnecessary.

The adaptive part $\boldsymbol{w}_{a}$ is continuously updated in order to remove any undesired signals other than that of at the looking direction from appearing at the array output. A blocking matrix $\boldsymbol{B}$ is first employed to block or prevent the desired signal at the looking direction from entering the adaptive part. Consequently, the input to the adaptive part mainly consists of the undesirable signals. The interference signal, after modifying by the adaptive weight vector $\boldsymbol{w}_{a}$, is then subtracted from the main beam in order to cancel the interference that is present in the main beam. This is achieved by minimizing the output energy of the beamformer using either the RLS or LMS adaptive filtering algorithms. Leakage of the desired signal through the adaptive part will lead to the annihilation or attenuation of the desired signal, i.e., signal cancellation. Robust beamforming techniques are usually employed to avoid this problem, which will be discussed later in Section V.

Denote the coefficients of the adaptive part by $\boldsymbol{w}_{i}$, $i=1, \ldots, N M_{B}$, as shown in the lower part of Fig. 7 . The array output of the GSC method can be written as

$$
y[n]=\boldsymbol{w}_{c}^{H} \boldsymbol{y}_{\mathrm{CPM} \_D}[n]-\boldsymbol{w}_{\boldsymbol{a}}^{H}[n] \boldsymbol{B} \boldsymbol{y}_{\mathrm{CPM}_{-} D}[n] .
$$

As mentioned in (3.8), the fixed weight part $\boldsymbol{w}_{c}$ is chosen to keep the beamformer response at the looking direction to be undistorted. This yields the following constraint:

$$
C^{H} \boldsymbol{w}_{c}=\mathbf{F}
$$

and $\boldsymbol{w}_{c}$ can be expressed as the following form:

$$
\boldsymbol{w}_{c}=C\left(\boldsymbol{C}^{H} \boldsymbol{C}\right)^{-1} \mathbf{F} .
$$

The adaptive weight vector $\boldsymbol{w}_{a}$ can be solved by minimizing the MSE function of the array output $y(n)$

$$
\boldsymbol{w}_{a_{-} L S}=\arg \min _{w_{a}} E\left[|y[n]|^{2}\right] \text {. }
$$

In the LMS algorithm, the weight vector $w_{a}$ is updated in the negative direction of the gradient of the MSE function $\nabla_{w_{a}} E\left[|y[n]|^{2}\right]$ as

$$
\boldsymbol{w}_{a}[n]=\boldsymbol{w}_{a}[n-1]+\mu \cdot y^{*}[n] \boldsymbol{B} \boldsymbol{y}_{\mathrm{CPM}_{-} D}[n]
$$

where $\mu$ is a stepsize parameter. It is called normalized LMS (NLMS) algorithm when $\mu$ is determined by $\tilde{\mu} /\left(\delta+\left\|\boldsymbol{y}_{\mathrm{CPM}_{-} D}\right\|^{2}\right)$, where $\tilde{\mu}$ is the normalized stepsize parameter and $\delta$ is a small positive constant to ensure $\widetilde{\mu}$ is not very large when the norm of the input is very small. Alternatively, if (3.11) is solved using the RLS algorithm, the weight vector is updated as [33]

$$
\boldsymbol{w}_{a}[n]=\boldsymbol{w}_{a}[n-1]+\boldsymbol{K}_{M} y^{*}[n]
$$

where

$$
\boldsymbol{K}_{M}[n]=\frac{\boldsymbol{P}[n-1] \boldsymbol{B} \boldsymbol{y}_{\mathrm{CPM}_{-} D}[n]}{\left(\lambda+\left(\boldsymbol{B} \boldsymbol{y}_{\mathrm{CPM}_{-} D}[n]\right)^{H} \boldsymbol{P}[n-1]\left(\boldsymbol{B} \boldsymbol{y}_{\mathrm{CPM}_{-} D}[n]\right)\right)}
$$

is the Kalman gain, $\boldsymbol{P}[n]$ is the inverse of the autocorrelation matrix and it can be updated as $\boldsymbol{P}[n]=(1 / \lambda)(\boldsymbol{P}[n-1]-$ $\left.\boldsymbol{K}_{M}[n]\left(\boldsymbol{B} \boldsymbol{y}_{\mathrm{CPM}_{-} D}[n]\right)^{H} \boldsymbol{P}[n-1]\right)$ with $\boldsymbol{P}[0]=\left(1 / \delta^{2}\right) \mathbf{I}, \delta^{2}$ is a small number to ensure that $\boldsymbol{P}[0]$ is nonsingular initially and $\lambda$ is the forgetting factor that controls the tracking ability and steady-state error of the RLS algorithm. Also, (3.11) can be solved using the $Q R$ decomposition algorithm.

Since the beamforming problem for UCCA is less studied in the literature than ULAs, we also summarize in the following, the implementation of a conventional tapped delay line-based broadband adaptive beamformer using the UCCA, i.e., without the compensation network. The geometry of the conventional UCCA is the same as the one shown in Fig. 1. The signal obtained at the $k$ th sensor has a phase difference of $\hat{r}_{p} \alpha \sin \theta \cos \left(\phi_{d}-\phi_{k}\right)$ relative to the one at the origin. The signal on the $k$ th sensor is delayed by $-\hat{r}_{p} \alpha \sin \theta \cos \left(\phi_{d}-\phi_{k}\right)$ to get the aligned signal. These broadband fractional delays can be realized using fractional delay digital filters, which in turn can be realized using FIR filters and designed by SOC [34]. The structures of the SMI and the GSC methods of traditional UCCA beamformers are analogous to those of the frequency invariant UCCA beamformer. The difference is that the input of the traditional UCCA beamformer is the aligned signals $x[n]$, while the input of the frequency invariant beamformer is the compensated phase mode signals $\boldsymbol{y}_{\mathrm{CPM}_{-} D}[n]$. The aligned signals are chosen in such a way that the signal at the desired angle is summed constructively while interference signals arriving at other angles will be suppressed [31]. The structures of these two traditional beamformers with $K$ sensors are shown in Figs. 8 and 9.

Similar to the one employing UCCA-FIB, the beamforming problem can be formulated as

$$
\text { minimize } \boldsymbol{w}^{H} \boldsymbol{R}_{x} \boldsymbol{w} \quad \text { subject to } \boldsymbol{C}^{H} \boldsymbol{w}=\mathbf{F}
$$




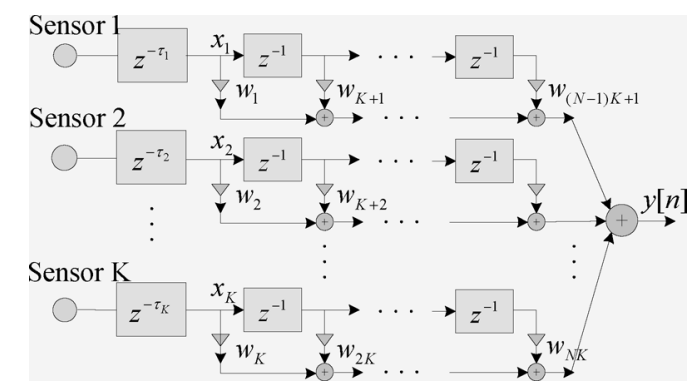

Fig. 8. UCCA beamformer using the SMI method and tapped-delay lines.

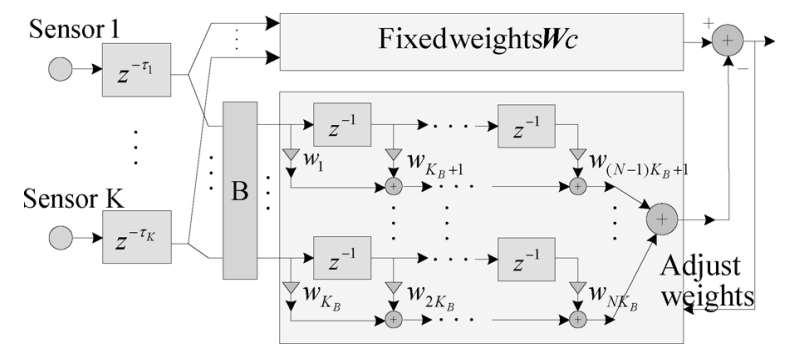

Fig. 9. UCCA beamformer using the GSC method and tapped-delay lines.

where

$$
C=\left[\begin{array}{lll}
1 & & 0 \\
& \ddots & \\
0 & & 1
\end{array}\right]
$$

is an $N K \times N$ matrix, $1=[1, \ldots, 1]^{T}$ is a $K \times 1$ constant vector, $\boldsymbol{R}_{x}=E\left\{\boldsymbol{x}[n] \cdot \boldsymbol{x}^{H}[n]\right\}, \boldsymbol{x}[n]$ consists of the aligned signals and their delayed versions, and $\mathbf{F}=[1,0, \ldots, 0]^{T}$ is a $N \times 1$ constant vector. Again, the optimal solution of the weight vector can be solved by the method of Langrage multipliers and it is given by

$$
\boldsymbol{w}_{\mathrm{opt}}=\boldsymbol{R}_{x}^{-1} \boldsymbol{C}\left(\boldsymbol{C}^{H} \boldsymbol{R}_{x}^{-1} \boldsymbol{C}\right)^{-1} \boldsymbol{F} \text {. }
$$

For the GSC method with the LMS algorithm, the weight vectors can be obtained as

$$
\begin{aligned}
\boldsymbol{w}_{c} & =\boldsymbol{C}\left(\boldsymbol{C}^{H} \boldsymbol{C}\right)^{-1} \mathbf{F} \\
\boldsymbol{w}_{a}[n] & =\boldsymbol{w}_{a}[n-1]+\mu \cdot y^{*}[n] \boldsymbol{B} \boldsymbol{x}[n] .
\end{aligned}
$$

We now present the simulation results for the various UCCA beamformers.

1) Example 2: Adaptive Beamforming Using UCCA-FIB: In this example, the UCCA-FIB is used to reconstruct the desired signal from $I$ interfering signals arriving at the array. The UCCA-FIB used in this example is the same as the two-ring UCCA in example 1 of Section II except that the interested frequency bandwidth is $[0.2 \pi, 0.65 \pi]$. Assume there are two noncoherent signals impinging on the array at angles $0^{\circ}$ and $50^{\circ}$, respectively. Here, we assume that the desired signal arrives the array at angle $0^{\circ}$ and the interference signal impinges on the

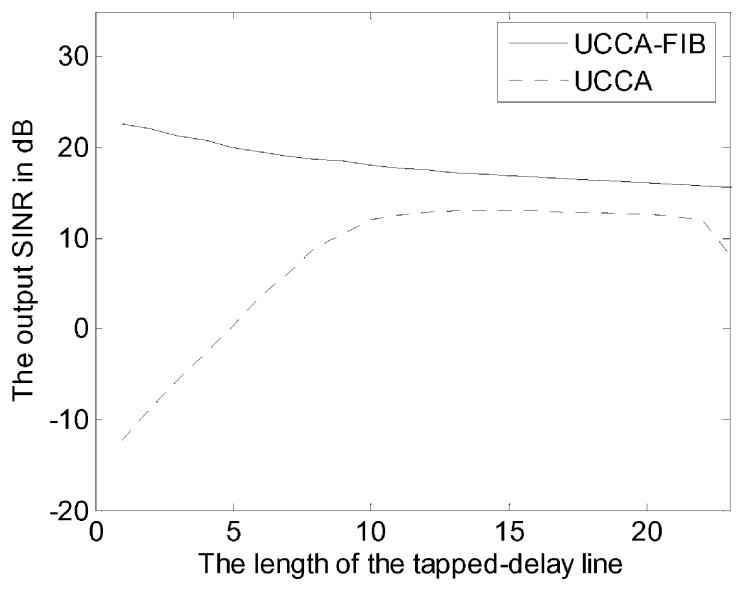

Fig. 10. Output SINRs of the two beamformers.

array at angle $50^{\circ}$. If the desired signal arrives the array at an angle other than $0^{\circ}$, the array can be electronically steered to it. Hence, the DOA of the desired signal can be assumed to be $0^{\circ}$. In this example, the desired signal is composed of 53 sinusoidal signals with frequencies ranging from $0.8 \times 10^{8}$ to $6 \times 10^{8} \mathrm{~Hz}$ at an interval of $0.1 \times 10^{8} \mathrm{~Hz}$. The interfering signal is also composed of 53 sinusoidal signals but with frequencies ranging from $0.83 \times 10^{8}$ to $6.3 \times 10^{8} \mathrm{~Hz}$ at an interval of $0.1 \times 10^{8} \mathrm{~Hz}$. The additive white Gaussian noise at each sensor is assumed to have the same power. The SINR is $-20 \mathrm{~dB}$ and the number of the snapshots is $2 \times 10^{4}$.

The performance of the beamforming using MVB method described in this section is first evaluated. The solid line in Fig. 10 shows the output SINR of the arrays versus different number of taps per phase mode in the UCCA-FIB. It is obtained by averaging 100 independent trials. A broadband beamformer using the MVB principle and UCCA without the FI characteristic is also implemented here for comparison. The specification of the array, input signals and sensor noises are identical to those used in the UCCA-FIB. The dotted line in Fig. 10 shows the beamforming result using the traditional UCCA without FI characteristics. From Fig. 10, we can see that the performance of using one tap per phase mode in the UCCA-FIB is much better than that of the UCCA when the number of taps is less than 10. When the numbers of the taps increase further, the performances of these two arrays are comparable. In other words, the proposed UCCA-FIB requires much fewer variable taps than the UCCA and this in turns translates to lower complexity in adaptation and shorter convergence time as will be shown in next example. The performances of the UCCA-FIB and the conventional UCCA level off as the number of taps is further increased. This is because the correlation matrix is very ill-conditioned when its dimension is increased to a very large value.

We now evaluate the performance of the adaptive UCCA-FIB in real-time beamforming using the LMS-GSC and RLS-GSC algorithms described previously. The stepsize $\mu$ of the LMS algorithm is set to 0.1 . Fig. 11 plots the output MSE against the iteration number for the LMS-GSC algorithm. The simulation results are obtained with 100 independent trials. The line labeled "UCCA-FIB" shows the output MSE between the beamforming 


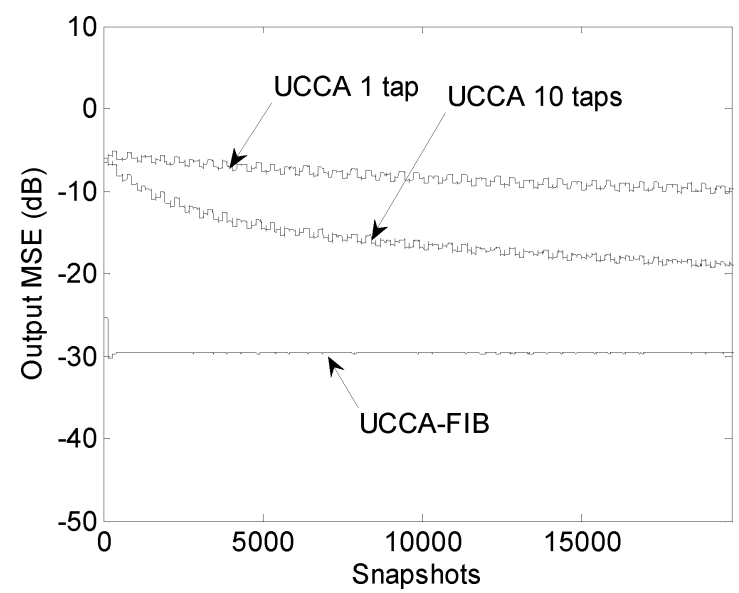

Fig. 11. Output errors of the two adaptive beamformers using the LMS-GSC algorithm.

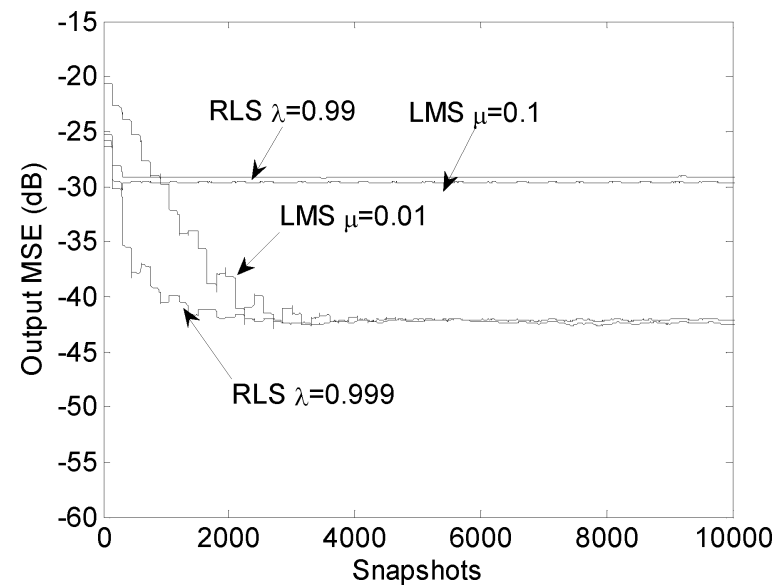

Fig. 12. Output errors of the adaptive UCCA-FIB with different algorithms and parameters.

output and the desired signal for the adaptive UCCA-FIB with one tap per phase mode. It converges approximately at the 800 snapshot and reaches a steady-state MSE of $-30 \mathrm{~dB}$.

For comparison, we also simulated the performance of a conventional beamformer using the UCCA without the compensation network. The number of tap per sensor element is one and the result is also shown in Fig. 11 with the line labeled UCCA 1 tap. From Fig. 11, we can see that the beamformer without the compensation network converges rather slowly. If the length of the tapped-delay is increased to 10, a lower higher output MSE is obtained, as shown in Fig. 11 with the line labeled UCCA 10 taps. It can be seen that its convergence speed is also much slower than the UCCA-FIB.

We now evaluate the performance of the UCCA-FIB using LMS-GSC and RLS-GSC algorithms with different parameters, both with one tap per phase mode. The simulation is shown in Fig. 12. As expected, the MSE decreases with increasing value of forgetting factors and decreasing value of stepsizes while the convergence speed exhibits an opposite dependency. It is also observed that the RLS algorithm convergences faster than the LMS algorithm for the same steady-state MSE error.

\section{DOA ESTIMATION AND RoBUST BEAMFORMING USING UCCA-FIB}

\section{A. DOA Estimation}

In some situations such as wireless communication and speech signal processing, the target may not be fixed. To obtain the desired signal, one can locate the target by DOA estimation and track its signal using the beamforming network. In this section, we briefly describe the DOA estimation algorithm [18] and evaluate the tracking performance of the proposed UCCA-FIB. A set of robust adaptive beamforming algorithms for the UCCA-FIB will also be developed and evaluated.

Broadband DOA estimation using frequency-invariant linear arrays has been studied in [8]. It can be viewed as a beamspace approach, where the outputs from a set of fixed beamformers, each having a different looking direction, is used to determine the DOA. This technique is also suitable to the proposed UCCAFIBs. In particular, a set of fixed UCCA FIBs with different looking directions are designed by modulating the weight vector of a UCCA-FIB with looking direction at the zero degree. Then, the MUSIC algorithm is applied to its outputs to estimate the DOAs of the broadband sources. Here, we only briefly summarize the DOA estimation method and the detail derivation can be found in a companion paper [18].

Consider $I$ broadband signals with frequency response $S(\omega)=\left[S_{1}(\omega), \ldots, S_{I}(\omega)\right]^{T}$ impinge a $P$-ring UCCA, respectively, at azimuth angles $\phi_{i}, i=1, \ldots, I$. According to (3.1), the frequency response of the beamformer output can be written as

$$
Y(\omega)=\boldsymbol{G}^{T}(\omega, \Phi) \boldsymbol{S}(\omega)+N(\omega)
$$

where $\boldsymbol{G}_{i}(\omega, \Phi)=\left[G_{i}\left(\omega, \phi_{1}\right), \ldots, G_{i}\left(\omega, \phi_{I}\right)\right]^{T}$, with $G_{i}\left(\omega, \phi_{i}\right)=\sum_{m=-L_{P}}^{L_{P}} g_{m, i} a_{G_{m, i}}\left(\omega, \phi_{i}\right), i=1, \ldots, I$, and $N(\omega)=\sum_{m=-L_{p}}^{L_{P}} g_{m} N_{m}(\omega)$. To estimate the DOA's, we use $J$ such beamformers to uniformly cover a given angular sector $\Delta \theta$, where the sources are assumed to lie in. Denote the output of these $J$ beamformers by $\boldsymbol{Y}_{F B_{-} 1}(\omega), \ldots, Y_{F B_{-} J}(\omega)$, and stack them together, one gets from (4.1) the following:

$$
\begin{aligned}
\boldsymbol{Y}_{F B}(\omega) & =\left[\boldsymbol{Y}_{F B_{-} 1}(\omega), \ldots, \boldsymbol{Y}_{F B_{-} J}(\omega)\right]^{T} \\
& =\left(\begin{array}{c}
\boldsymbol{G}_{1}(\omega, \Phi) \\
\vdots \\
\boldsymbol{G}_{J}(\omega, \Phi)
\end{array}\right) \boldsymbol{S}(\omega)+\left(\begin{array}{c}
N^{(1)}(\omega) \\
\vdots \\
N^{(J)}(\omega)
\end{array}\right) \\
& =\boldsymbol{G}_{F B}(\omega, \Phi) \boldsymbol{S}(\omega)+N_{F B}(\omega)
\end{aligned}
$$

where $\boldsymbol{G}_{i}(\omega, \Phi)$ are the gain of the signal in (4.1) and $N^{(i)}(\omega)$ are noise for the $i$ th beamformer. Taking the IDFT of (4.2), one gets the beamformer output in time domain as follows:

$$
\boldsymbol{y}_{F B}(n)=\left[\boldsymbol{y}_{F B_{-} 1}(n), \ldots, \boldsymbol{y}_{F B_{-} J}(n)\right]^{T}
$$

and the following covariance matrix of $\boldsymbol{y}_{F B}(n)$ :

$$
\boldsymbol{R}_{y}(\Phi)=\sum_{n=-\infty}^{\infty} \boldsymbol{y}_{F B}(n) \boldsymbol{y}_{F B}^{H}(n) .
$$


Since $G(\omega, \Phi)$ has been designed to be approximately frequency invariant in the interested band, we have $\mathbf{G}(\omega, \Phi) \approx$ $\mathbf{G}(\Phi), \forall \omega \in\left[\omega_{l}, \omega_{u}\right]$. Following the derivation in [8], the DOAs of the sources can be estimated using the beamformer outputs $\boldsymbol{y}_{F B}(n)$ by computing the following MUSIC spectrum:

$$
\Theta(\phi)=\frac{\mathbf{G}(\phi)^{H} \mathbf{G}(\phi)}{\mathbf{G}^{H}(\phi) \mathbf{E}_{N} \mathbf{E}_{N}^{H} \mathbf{G}(\phi)}
$$

where $\mathbf{G}(\phi)$ is the array response at angle $\phi$ in the interested band and $\mathbf{E}_{N}$ is the eigen-vector of the noise subspace that can be obtained from $R_{y}(\Phi)$ by generalized eigen-decompostion.

\section{B. Robust Beamforming}

As mentioned earlier, due to DOA estimation and other implementation errors, the actual array response may derivate from the assumed signal model. As a result, conventional beamforming methods such as GSC may suppress the desired signal as well as the interfering signals. Robust beamforming algorithms were required to remedy this problem.

In the method of diagonal loading [22], the norm of the weight vector is constrained in order to avoid severe signal cancellation. Adopting this idea in our UCCA-FIB yields the following robust adaptive beamforming problem:

$$
\begin{aligned}
& \operatorname{minimize} \boldsymbol{w}^{H} \boldsymbol{R}_{y_{-} \mathrm{CPM}} \boldsymbol{w} \\
& \text { subject to } \boldsymbol{C}^{H} \boldsymbol{w}=\mathbf{F}, \boldsymbol{w}^{H} \boldsymbol{w} \leq \delta^{2}
\end{aligned}
$$

where $\delta$ is a constant norm bound for the weight vector. Using the Lagrange multiplier technique, the analytical solution to this problem can be written as

$$
\boldsymbol{w}_{\mathrm{opt}}=\left(\boldsymbol{R}_{y_{-} \mathrm{CPM}}+\varepsilon \mathbf{I}\right)^{-1} \boldsymbol{C}\left(\boldsymbol{C}^{H}\left(\boldsymbol{R}_{y_{-} \mathrm{CPM}}+\varepsilon \mathbf{I}\right)^{-1} \boldsymbol{C}\right)^{-1} \mathbf{F}
$$

where $\varepsilon$ is the Lagrange multiplier or loading factor. In practical implementation, it is sometimes difficult to set the value of $\varepsilon$ to satisfy the norm constraint. In [22], Cox proposed a scaled projection (SP) method for updating the weight vector directly from $\delta^{2}$. This algorithm can also be applied to the UCCA-FIB and the resulting algorithm is given in the following.

For the LMS-GSC algorithm, the fixed weight vector $\boldsymbol{w}_{c}$ does not depend on the covariance matrix of the beamformer input, and it has the same solution as in (3.10). The adaptive part $w_{a}$ is adapted with the following scaled projection algorithm [22]:

$$
\boldsymbol{w}_{a}[n+1]= \begin{cases}\tilde{\boldsymbol{w}}_{a}[n+1], & \text { for }\left\|\tilde{\boldsymbol{w}}_{a}[n+1]\right\|^{2} \leq b^{2} \\ \frac{b \tilde{\boldsymbol{w}}_{a}[n+1]}{\left\|\tilde{\boldsymbol{w}}_{a}[n+1]\right\|_{2}}, & \text { for }\left\|\tilde{\boldsymbol{w}}_{a}[n+1]\right\|^{2}>b^{2} .\end{cases}
$$

The SP method makes the LMS updating simple and effective under the quadratic constraint in (4.6), however, it does not appear effective for RLS algorithm due to the difficulty in choosing the diagonal loading term. Recently, Z. Tian et al. proposed a variable loading method for the RLS algorithm under quadratic constraint [35]. In this method, the update in (3.12) is first treated as a tentative updating

$$
\tilde{\boldsymbol{w}}_{a}[n]=\boldsymbol{w}_{a}[n-1]+\boldsymbol{K}_{M} y^{*}(n) .
$$

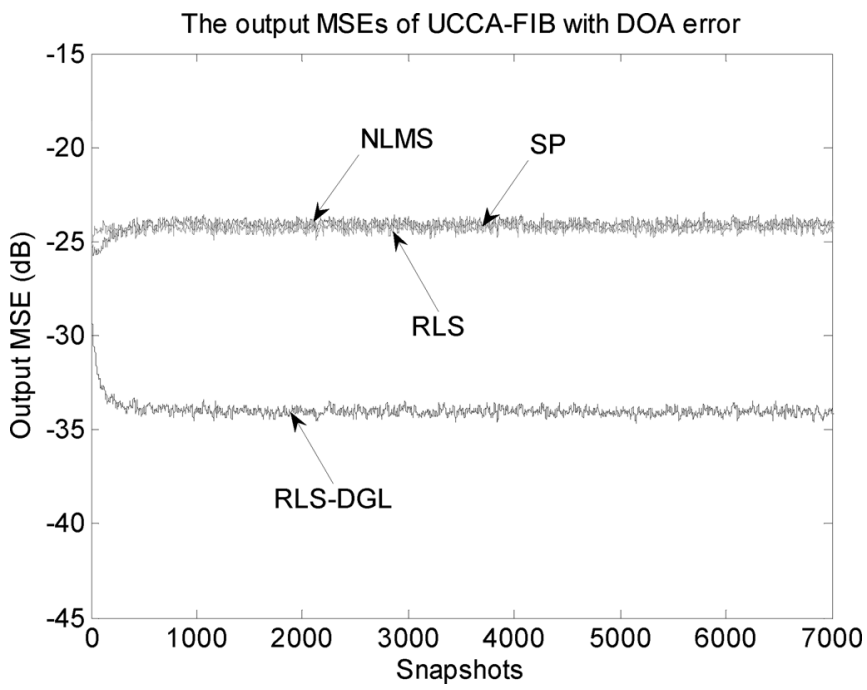

Fig. 13. MSE results of the various adaptive UCCA-FIB algorithms with DOA estimation error.

The weight vector is further modified as follows [35]:

$$
\begin{cases}\boldsymbol{w}_{a}[n]=\tilde{\boldsymbol{w}}_{a}[n], & \text { for }\left\|\tilde{\boldsymbol{w}}_{a}[n]\right\|^{2} \leq \delta^{2} \\ \boldsymbol{w}_{a}[n]=\tilde{\boldsymbol{w}}_{a}[n]-\lambda[n] \boldsymbol{v}_{a}[n], & \text { for }\left\|\tilde{\boldsymbol{w}}_{a}[n]\right\|^{2}>\delta^{2}\end{cases}
$$

where $\boldsymbol{v}_{a}[n]=\boldsymbol{P}[n] \tilde{\boldsymbol{w}}_{a}[n], \lambda[n]=\left(-b-\operatorname{Re}\left\{\sqrt{b^{2}-4 a c}\right\}\right) /$ $2 a, a=\left\|\boldsymbol{v}_{a}[n]\right\|^{2}, b=-2 \operatorname{Re}\left\{\boldsymbol{v}_{a}^{H}[n] \tilde{\boldsymbol{w}}_{a}[n]\right\}, c=\left\|\tilde{\boldsymbol{w}}_{a}[n]\right\|^{2}-$ $\delta^{2}$, and $\operatorname{Re}\{\cdot\}$ means real part.

1) Example 3: Robust Beamforming Using UCCA-FIB: In this example, the target is assumed to be moving to evaluate the tracking ability of the proposed UCCA-FIB. In addition, DOA and calibration errors are included to evaluate the effectiveness of the robust beamforming techniques for the UCCA-FIB.

DOA Errors: The angle of arrival (AOA) of the desired signal and the interference are assumed to be $0^{\circ}$ and $50^{\circ}$, respectively. The desired signal and interference signal are assumed to be Gaussian. The performance of multisinusoidal signals is comparable to that of Gaussian signal. The result is omitted here due to page limitation. The SNR is set to $20 \mathrm{~dB}$ and the SINR is $-14 \mathrm{~dB}$. The normalized stepsize $\widetilde{\mu}$ and the forgetting vector $\lambda$ are, respectively, set to 0.8 and 0.999 . Fig. 13 shows the beamforming results when the DOA estimation error of the desired signal is $5^{\circ}$. Significant signal cancellation occurs in the RLS and NLMS algorithms. With diagonal loading (DGL), the beampattern is broadened and the desired signal is better preserved. However, there is no performance improvement in the SP algorithm, the output MSE of the SP algorithm is almost identical to NLMS algorithm. The simulation results are obtained by averaging 200 independent trials and the MSE curves are obtained by temporal smoothing using a window size of 10 samples.

Calibration Errors: For simplicity, it is assumed that there is no sensor error in the elevation angle $\theta$. The crosses and circles in Fig. 14 denote, respectively, the ideal and actual locations of the sensors. The deviation is generated randomly using a white Gaussian distribution with a variance of 0.25 . The signal model is the same as in the first part of this example. The normalized stepsize $\widetilde{\mu}$ and the forgetting vector $\lambda$ are, respectively, set to 0.03 and 0.999 . Fig. 15 shows the MSE results 


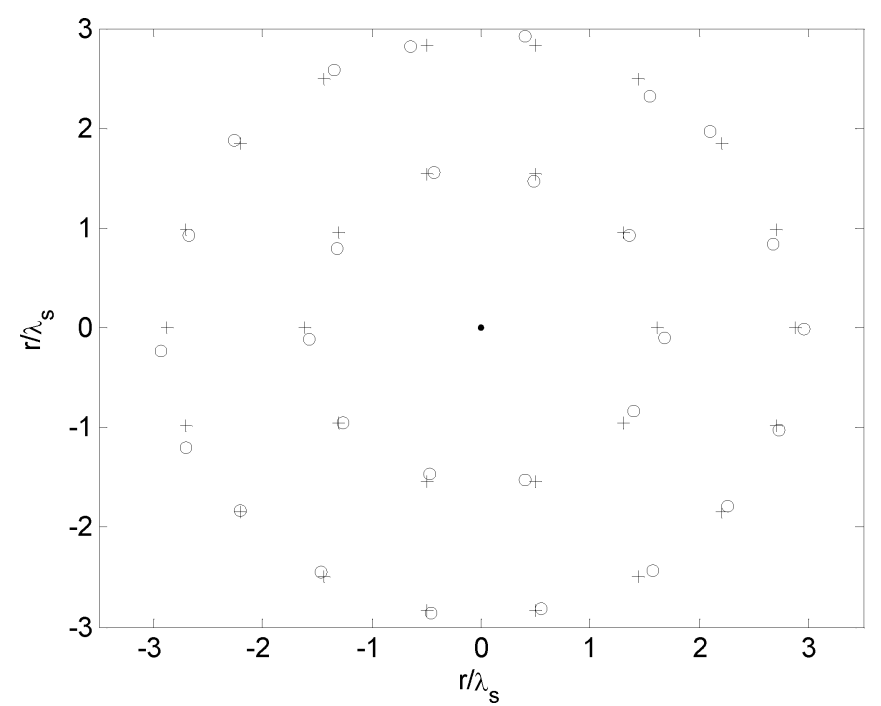

Fig. 14. Ideal and actual locations of the sensors.

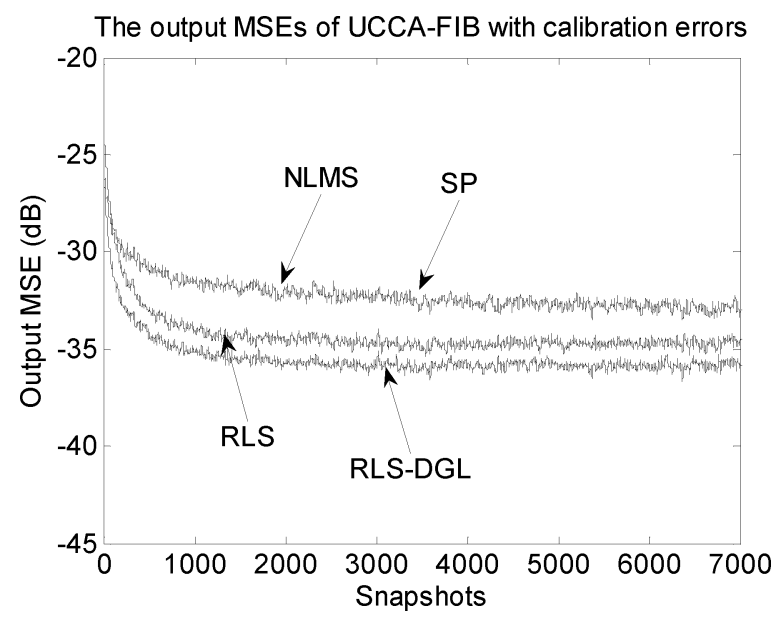

Fig. 15. MSE results of the adaptive UCCA-FIB algorithms with calibration errors.

for the UCCA-FIB adaptive algorithms. Due to the diagonal loading, the RLS-DGL algorithm better preserves the desired signal than the RLS algorithm. The NLMS and SP algorithms converge slower than the RLS-DGL algorithm and they yield higher steady-state errors.

Tracking Performance: The third case is on tracking a moving target using UCCA-FIB. The DOA of the target is assumed to change at every interval of 4000 snapshots, and its values are $\left[0^{\circ}, 1^{\circ}, 2^{\circ}, 3^{\circ}, 4^{\circ}\right]$, respectively. The angles of the interference are varied in the same way and the angles are $\left[50^{\circ}, 51^{\circ}, 52^{\circ}, 53^{\circ}, 54^{\circ}\right]$. The signal model is set to the same as the one in the first part of this example. The lines labeled NLMS and SP in Fig. 16 are the tracking results that employ the NLMS-GSC and NLMS algorithms with scaled projection, respectively. Due to the robust characteristic of the UCCA-FIB, these two methods have almost the same performance. The MSEs converge after several hundreds of snapshots. The lines labeled "RLS" and "RLS-DGL" are, respectively, the tracking results for the RLS algorithm and RLS algorithm with diagonal

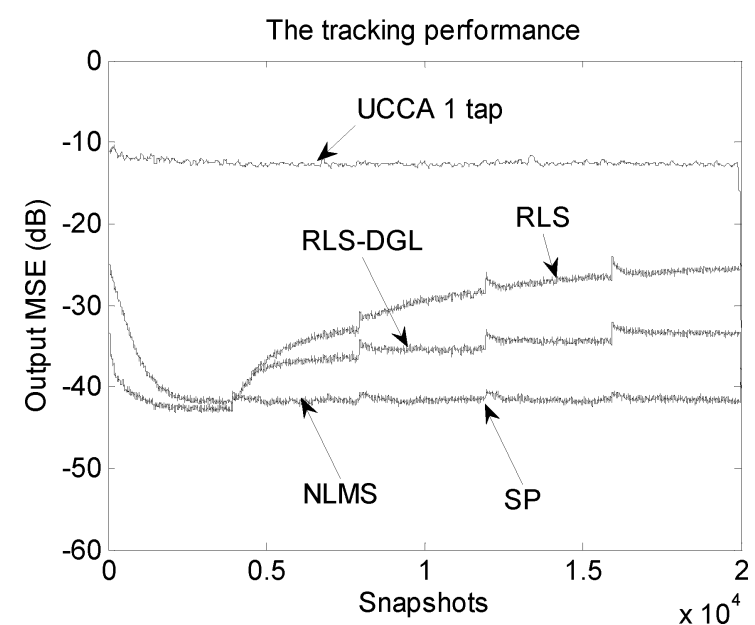

Fig. 16. Tracking performances of the various UCCA beamforming algorithms.

loading. They have a faster initial convergence speed than the NLMS algorithms. However, the NLMS algorithm tracks the moving target better than the RLS and RLS-DGL algorithms. Due to diagonal loading, RLS-DGL method tracks the target better than the RLS algorithm. The tracking result using a UCCA without the compensation network and with one tap per sensor is also shown in Fig. 16 as the line labeled "UCCA 1 tap". It can be seen that the tracking results are unsatisfactory. Since the target is varying every 4000 snapshots, it is unable to follow the moving target, unlike its frequency invariant counterpart.

Before drawing the conclusions, we now roughly compare the arithmetic complexity of the adaptive beamformers using UCCA FIB and the one employing conventional UCCA without compensation filters. The arithmetic complexity of a digital beamformer usually consists of the complexities for the fixed filtering and the adaptive filtering parts. The order of the arithmetic complexity per sample for the fixed filtering part is usually linear in the filter length, while the order of arithmetic complexity per unit time for the adaptive filtering part will depend on the algorithm used. Let $N$ denote the number of adaptive coefficients, if SMI algorithm is used, the arithmetic complexity per unit time is of order $O\left(N^{3}\right)$. If the blocking matrix $\boldsymbol{B}$ has dimension of $N_{B} \times N_{y}$, the arithmetic complexities per unit time for LMS-GSC and RLS-GSC are of order $O\left(2 N+N_{B} \times N_{y}\right)$ and $O\left(4 N^{2}+5 N+N_{B} \times N_{y}\right)$, respectively. In robust beamforming, the arithmetic complexity for the scaled projection LMS and diagonal loading RLS algorithms are of order $O\left(4 N+N_{B} \times N_{y}\right)$ and $O\left(5 N^{2}+10 N+N_{B} \times N_{y}\right)$, respectively. For example, in RLS-GSC, let $T$ denote the length of the adaptive tapped-delay line and $L_{1}$ denote the length of the compensation filters in a UCCA FIB, the complexity of the fixed and adaptive parts are, respectively, $O\left(\left(\sum_{p=1}^{P} M_{p}\right) \times L_{1}\right)$ and $O\left(4\left[\left(M_{P}-1\right) \times T\right]^{2}+5\left[\left(M_{P}-1\right) \times T\right]+\left(M_{P}-1\right) \times M_{P}\right)$, where $M_{p}, p=1, \ldots P$, is the number of the usable phase modes of the $p$ th ring and the size of the blocking matrix $B$ is $\left(M_{P}-1\right) \times M_{P}$. Let $L_{2}$ denote the length of the broad band fractional delay filters used in the traditional UCCA, 
TABLE I

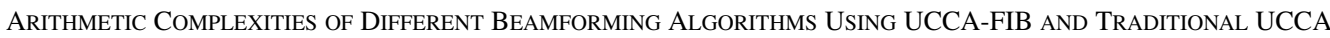

\begin{tabular}{|l|l|l|l|l|}
\hline \multirow{2}{*}{ Algorithms } & \multicolumn{2}{|c|}{ UCCA-FIB } & \multicolumn{2}{c|}{ Traditional UCCA } \\
\cline { 2 - 5 } & Arithmetic complexity & $\begin{array}{l}\text { Numerical } \\
\text { example }\end{array}$ & Arithmetic complexity & 3388 \\
\hline $\begin{array}{l}\text { Fixed filtering } \\
O(N)\end{array}$ & $O\left(L_{1} \times \sum_{p=1}^{P} M_{p}\right)$ & 558 & $O\left(L_{2} \times \sum_{p=1}^{P} K_{p}\right)$ & 21952 \\
\hline $\begin{array}{l}\text { SMI } \\
O\left(N^{3}\right)\end{array}$ & $O\left(\left(M_{P} \times T\right)^{3}\right)$ & 729 & $O\left(\left(\sum_{p=1}^{P} K_{p} \times T\right)^{3}\right)$ & 810 \\
\hline $\begin{array}{l}\text { LMS } \\
O\left(2 N+N_{\mathrm{B}} \times N_{\mathrm{y}}\right)\end{array}$ & $O\left(\begin{array}{l}2\left(M_{P}-1\right) T+ \\
\left(M_{P}-1\right) \times M_{P}\end{array}\right)$ & 88 & $O\left(\begin{array}{l}2\left[\left(\sum_{p=1}^{P} K_{p}-1\right) \times T\right]+ \\
\left(\sum_{p=1}^{P} K_{p}-1\right) \times \sum_{p=1}^{P} K_{p}\end{array}\right)$ & \\
\hline $\begin{array}{l}\text { RLS } \\
O\left(4 N^{2}+5 N+N_{B} \times N_{y}\right)\end{array}$ & $O\left(\begin{array}{l}4\left[\left(M_{P}-1\right) T\right]^{2}+ \\
5\left(M_{P}-1\right) T+\left(M_{P}-1\right) \times M_{P}\end{array}\right)$ & 368 & $\left.\begin{array}{l}4\left[\left(\sum_{p=1}^{P} K_{p}-1\right) T\right]^{2}+5\left[\left(\sum_{p=1}^{P} K_{p}\right.\right. \\
-1) T]+\left(\sum_{p=1}^{P} K_{p}-1\right) \times \sum_{p=1}^{P} K_{p}\end{array}\right)$ & 3807 \\
\hline $\begin{array}{l}\text { SP } \\
O\left(4 N+N_{\mathrm{B}} \times N_{\mathrm{y}}\right)\end{array}$ & $O\left(\begin{array}{l}4\left(M_{P}-1\right) T+ \\
\left(M_{P}-1\right) \times M_{P}\end{array}\right)$ & 104 & $O\left(\begin{array}{l}4\left[\left(\sum_{p=1}^{P} K_{p}-1\right) \times T\right]+ \\
\left(\sum_{p=1}^{P} K_{p}-1\right) \times \sum_{p=1}^{P} K_{p}\end{array}\right)$ & 864 \\
\hline $\begin{array}{l}\text { RLS-DGL } \\
O\left(5 N^{2}+10 N+N_{B} \times N_{y}\right)\end{array}$ & $O\left(\begin{array}{l}5\left[\left(M_{P}-1\right) T\right]^{2}+ \\
10\left(M_{P}-1\right) T+\left(M_{P}-1\right) \times M_{P}\end{array}\right)$ & & $O\left(\begin{array}{l}5\left[\left(\sum_{p=1}^{P} K_{p}-1\right) T\right]^{2}+10\left[\left(\sum_{p=1}^{P} K_{p}\right.\right. \\
-1) T]+\left(\sum_{p=1}^{P} K_{p}-1\right) \times \sum_{p=1}^{P} K_{p}\end{array}\right)$ & 4761 \\
\hline
\end{tabular}

${ }^{*} N$ is the number of the coefficients, $T$ is the length of the tapped-delay line and the size of the blocking matrix is $N_{\mathrm{B}} \times N_{y}$. In the UCCA-FIB, $L_{1}$ is the length of the compensation filters, $M_{\mathrm{p}}, p=1, \ldots, P$, is the number of the usable phase modes in the $p^{\text {th }}$ ring. In traditional UCCA, $L_{2}$ is the length of the broadband fractional delay filter and $K_{\mathrm{p}}, p=1, \ldots, P$, is the number of the sensors in the $p^{\text {th }}$ ring. In the numerical examples, the parameter setting is the same as the ones in Example 1 and Example 2, i.e. $T=1, L_{1}=31$, $L_{2}=121, M_{1}=M_{2}=9, K_{1}=10, K_{2}=18, N_{\mathrm{B}} \times N_{y}$ is $8 \times 9$ and $27 \times 28$ in UCCA-FIB and UCCA, respectively. SP denotes the scaled projection LMS algorithm and RLS-DGL denotes the RLS algorithm with diagonal loading.

the complexity of the fixed and adaptive filtering parts are, respectively, given by $O\left(\left(\sum_{p=1}^{P} K_{p}\right) \times L_{2}\right)$ and

$$
\begin{aligned}
O\left(4\left[\left(\sum_{p=1}^{P} K_{p}-1\right) \times T\right]^{2}+\right. & 5\left[\left(\sum_{p=1}^{P} K_{p}-1\right) \times T\right] \\
& \left.+\left(\sum_{p=1}^{P} K_{p}-1\right) \times \sum_{p=1}^{P} K_{p}\right)
\end{aligned}
$$

where $K_{p}, p=1, \ldots, P$, is the number of the sensors in the $p$ th ring and the size of the blocking matrix is $\left(\sum_{p=1}^{P} K_{p}-\right.$ 1) $\times \sum_{p=1}^{P} K_{p}$. In the simulation of this example, the numbers of sensors in the two rings of the UCCA are 10 and 18 , respectively. The length $\left(L_{1}\right)$ of the compensation filters required is 31 and the number of usable phase modes is 9 . On the other hand, the length $\left(L_{2}\right)$ of the broadband fractional delay filters used in the traditional UCCA is 121 . Therefore, the arithmetic complexity of this UCCA-FIB is $O(558)+O\left(256 T^{2}+40 T+72\right)$, while that of a traditional UCCA is $O(3388)+O\left(2916 T^{2}+\right.$ $135 T+756)$. In addition, the output signal to interference plus noise ratio (SINR) of the UCCA-FIB with $T=1$ is better than that of the traditional UCCA with $T=10$ for broadband Gaussian as well as multisinusoidal inputs tested. Therefore, the proposed UCCA-FIB is a good alternative to traditional UCCAs because of its lower arithmetic and implementation complexities, higher output SINR and faster convergence speed. The comparison between the arithmetic complexities of the proposed UCCA-FIB and the traditional UCCA is summarized in Table I.

\section{CONCLUSION}

New recursive adaptive beamforming algorithms for a class of uniform concentric circular arrays with frequency invariant characteristics using MVDR and GSC methods are presented. The proposed adaptive UCCA-FIB is obtained by compensating the frequency dependency of individual phase modes using a digital beamforming network and it involves a small set of adaptive coefficients. Robust versions of these recursive beamforming algorithms to mitigate the adverse effect of DOA and sensor location errors are presented. The DOA estimation and target tracking methods based on the UCCA-FIB are also proposed. Computer simulation results are presented to illustrate the performance of the proposed methods. It was found that the new adaptive UCCA-FIB offers improved convergence speed, arithmetic complexity and steady-state error than conventional broadband beamformers without employing the compensation network.

\section{REFERENCES}

[1] D. H. Johnson and D. E. Dudgeon, Array Signal Processing: Concepts and Techniques. Englewood Cliffs, NJ: Prentice-Hall, 1993.

[2] H. Krim and M. Viberg, "Two decades of array signal processing research: The parametric approach," IEEE Signal Process. Mag., vol. 13, no. 4, pp. 67-94, Jul. 1996.

[3] B. D. Van Veen and K. M. Buckley, "Beamforming: A versatile approach to spatial filtering," IEEE ASSP Mag., vol. 52, no. 2, pp. 4-24, Apr. 1988.

[4] K. Nishikawa, T. Yamamoto, K. Oto, and T. Kanamori, "Wideband beamforming using fan filter," in Proc. IEEE ISCAS, 1992, pp. 533-536.

[5] T. Sekiguchi and Y. Karasawa, "Wideband beamspace adaptive array utilizing FIR Fan filters for multibeam forming," IEEE Trans. Signal Process., vol. 48, no. 1, pp. 277-284, Jan. 2000. 
[6] D. B. Ward, R. A. Kennedy, and R. C. Williamson, "Theory and design of broadband sensor arrays with frequency invariant far-field beam patterns," J. Acoust. Soc. Amer., vol. 97, no. 2, pp. 1023-1034, Feb. 1995.

[7] D. B. Ward, R. A. Kennedy, and R. C. Williamson, "FIR filter design for frequency invariant beamformers," IEEE Signal Process. Lett., vol. 3, no. 3, pp. 69-71, Mar. 2000.

[8] D. B. Ward, Z. Ding, and R. A. Kennedy, "Broadband DOA estimation using frequency invariant beamforming," IEEE Trans. Signal Process., vol. 46, no. 5, pp. 1463-1469, May 1998.

[9] L. J. Griffiths and C. W. Jim, "An alternative approach to linearly constrained adaptive beamforming," IEEE Trans. Ant. Propag., vol. AP-30, no. 1, pp. 27-34, Jan. 1982

[10] M. Ghavami and R. Kohno, "Recursive fan filters for a broad-band partially adaptive antenna," IEEE Trans. Commun., vol. 48, no. 2, pp. 185-188, Feb. 2000.

[11] R. O. Schmidt, "Multiple emitter location and signal parameter estimation," IEEE Trans. Ant. Propag., vol. AP-34, no. 3, pp. 276-280, Mar. 1986.

[12] T. Do-Hong, F. Demmel, and P. Russer, "A method for wideband direction-of-arrival estimation using frequency-domain frequency-invariant beamformers," in Proc. IEEE Int. Symp. Ant. Propag., 2003, pp. 244-247.

[13] S. C. Chan and K. S. Pun, "On the design of digital broadband beamformer for uniform circular array with frequency invariant characteristics," in Proc. IEEE ISCAS, 2002, pp. 693-696.

[14] H. Steyskal, "Circular array with frequency-invariant pattern," in Dig. IEEE Int. Symp. Ant. Propag., 1989, pp. 1477-1480.

[15] S. C. Chan and H. H. Chen, "Theory and design of uniform concentric circular arrays with frequency invariant characteristics," in Proc. IEEE ICASSP, 2005, pp. 805-808.

[16] S. C. Chan, H. H. Chen, and K. L. Ho, "Adaptive beamforming using uniform concentric circular arrays with frequency invariant characteristics," in Proc. IEEE ISCAS, 2005, pp. 4321-4324.

[17] T. Rahim and D. E. N. Davies, "Effect of directional elements on the directional response of circular antenna arrays," Proc. IEE, Part H: Microw., Opt. Ant., vol. 129, no. 1, pp. 18-22, Feb. 1982.

[18] S. C. Chan and H. H. Chen, "Uniform concentric circular arrays with frequency invariant characteristics-Theory, design, adaptive beamforming and DOA estimation," IEEE Trans. Signal Process., vol. 55, no. 1, pp. 165-177, Jan. 2007.

[19] C. P. Mathews and M. D. Zoltowski, "Eigenstructure techniques for 2-D angle estimation with uniform circular arrays," IEEE Trans. Signal Process., vol. 42, no. 9, pp. 2395-2407, Sep. 1994.

[20] J. Capon, "High-resolution frequency-wavenumber spectrum analysis," Proc. IEEE, vol. 57, no. 8, pp. 1408-1418, Aug. 1969.

[21] H. L. Van trees, Optimum Array Processing: Part IV of Detection, Estimation and Modulation Theory. New York: Wiley-Interscience, 2002.

[22] H. Cox, R. M. Zeskind, and M. H. Owen, "Robust adaptive beamforming," IEEE Trans. ASSP, vol. 35, no. 10, pp. 1365-1376, Oct. 1987.

[23] S. Vorobyov, A. B. Gershman, and Z. Q. Luo, "Robust adaptive beamforming using worst-case performance optimization: A solution to the signal mismatch problem," IEEE Trans. Signal Process., vol. 51, no. 2, pp. 313-324, Feb. 2003.

[24] R. Lorenz and S. P. Boyd, "Robust minimum variance beamforming," IEEE Trans. Signal Process., vol. 53, no. 5, pp. 1684-1696, May 2005.

[25] M. Abramowitz and I. A. Stegun, Handbook of Mathematical Functions. New York: Dover, 1965.

[26] T. W. Parks and J. H. McClellan, "Chebyshev approximation for nonrecursive digital filters with linear phase," IEEE Trans. Circuits Syst., vol. 19, no. 2, pp. 189-194, Mar. 1972.

[27] J. O. Coleman and D. P. Scholnik, "Design of nonlinear phase FIR filters with second-order cone programming," in Proc. IEEE MWSCAS, 1999, pp. 409-412.

[28] M. S. Lobo, L. Vandenberghe, S. Boyd, and H. Lebret, "Applications of second-order cone programming," Linear Algebra Appl., vol. 248, pp. 193-228, Nov. 1998.

[29] I. D. Longstaff, P. E. K. Chow, and D. E. N. Davies, "Directional properties of circular arrays," Proc. IEE, vol. 114, no. 6, pp. 713-718, 1967.

[30] M. Wax and J. Sheinvald, "Direction finding of coherent signals via spatial smoothing for uniform circular arrays," IEEE Trans. Ant. Propag., vol. 42, no. 5, pp. 613-620, May 1994.

[31] O. L. Frost, III, "An algorithm for linearly constrained adaptive array processing," Proc. IEEE, vol. 60, no. 8, pp. 926-935, Aug. 1972.

[32] K. M. Buckley and L. J. Griffiths, "An adaptive generalized sidelobe canceller with derivative constraints," IEEE Trans. Ant. Propag., vol. AP-34, no. 3, pp. 311-319, Mar. 1986.

[33] S. Haykin, Adaptive Filter Theory, 3rd ed. Upper Saddle River, NJ: Prentice-Hall, 1996.

[34] K. M. Tsui and S. C. Chan, "Design of FIR digital filters with prescribed flatness and peak error constraints using second order cone programming," IEEE Trans. Circuits Syst. II, Exp. Briefs, vol. 52, no. 8, pp. 601-605, Sep. 2005.

[35] Z. Tian, K. L. Bell, and H. L. Van Trees, "A recursive least squares implementation for LCMP beamforming under quadratic constraint," IEEE Trans. Signal Process., vol. 49, no. 6, pp. 1138-1145, Jun. 2001.

[36] J. F. Sturm, "Using sedumi 1.02, a MATLAB toolbox for optimization over symmetric cones," Optim. Meth. Softw., vol. 11-12, pp. 625-653, 1999.

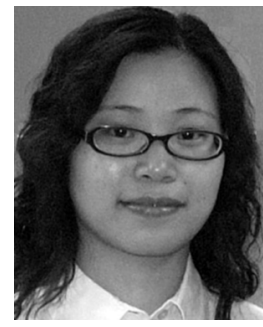

H. H. Chen (S'06) received the M.Eng. degree in electrical engineering from Nankai University, Tianjin, China, in 2002, and the Ph.D. degree from in electrical and electronic engineering from The University of Hong Kong, Hong Kong, in 2006.

In 2007, she joined the Communication System Group, Darmstadt University of Technology, Darmstadt, Germany, where she is currently working as a Postdoctoral Fellow. Her current research interests include robust adaptive beamforming, broadband sensor arrays, and their applications and statistical array signal processing.

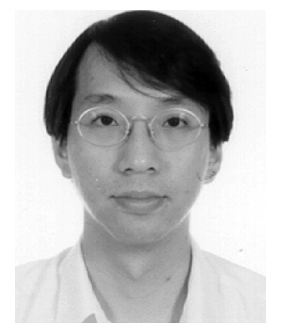

S. C. Chan (S'87-M'92) received the B.Sc. (Eng.) and Ph.D. degrees from the University of Hong Kong, Hong Kong, in 1986 and 1992, respectively.

Since 1994, he has been with the Department of Electrical and Electronic Engineering, the University of Hong Kong, where he is currently an Associate Professor. In 1990, he joined City Polytechnic of Hong Kong, Hong Kong, as an Assistant Lecturer and later as a University Lecturer. He was a Visiting Researcher at Microsoft Corporation, Redmond, WA, in 1998 and at Microsoft China, Beijing, China, in 1999. His research interests include fast transform algorithms, filter design and realization, multirate signal processing, communications signal processing, and image-based rendering.

Dr. Chan is currently a member of the Digital Signal Processing Technical Committee of the IEEE Circuits and Systems Society. He was Chairman of the IEEE Hong Kong Chapter of Signal Processing from 2000 to 2002.

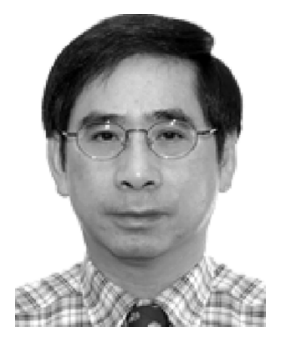

Member of HKIE.
K. L. Ho (M'78-SM'04) received the B.Sc (Eng.) and the M.Phil. degrees in electrical engineering from the University of Hong Kong, Hong Kong, in 1971 and 1973, respectively, and the Ph.D degree from the University of London, London, U.K., in 1977.

In 1984, he joined the Department of Electrical and Electronic Engineering, the University of Hong Kong. His current research interests include signal processing and communications systems.

Dr. Ho is a Chartered Engineer of the Engineering Council, U.K., a Fellow of the IEE, U.K., and 